\title{
The Quantum Internet
}

\author{
H. J. Kimble \\ Norman Bridge Laboratory of Physics 12-33 \\ California Institute of Technology \\ Pasadena, California 91125, USA
}

(Dated: June 25, 2008)

\begin{abstract}
Quantum networks offer a unifying set of opportunities and challenges across exciting intellectual and technical frontiers, including for quantum computation, communication, and metrology. The realization of quantum networks composed of many nodes and channels requires new scientific capabilities for the generation and characterization of quantum coherence and entanglement. Fundamental to this endeavor are quantum interconnects that convert quantum states from one physical system to those of another in a reversible fashion. Such quantum connectivity for networks can be achieved by optical interactions of single photons and atoms, thereby enabling entanglement distribution and quantum teleportation between nodes.
\end{abstract}

\section{INTRODUCTION}

The past two decades have witnessed a wide range of fundamental discoveries in quantum information science (QIS), ranging from a quantum algorithm that places public-key cryptography at risk to a protocol for the teleportation of quantum states [1. This union of quantum mechanics and information science has fostered great advances in our understanding of the quantum world and in our ability to control coherently individual quantum systems [2]. Unique capabilities of quantum systems to process and distribute information have been identified, and powerful new perspectives for understanding the complexity and subtleties of quantum dynamical phenomena have emerged.

Within the broad context of QIS, quantum networks play an important role, both for the formal analysis and the physical implementation of quantum computation, communication, and metrology [2, 3, 4, 5, 6]. Figure 1)(a) illustrates a notional quantum network based upon the original proposals in Refs. 4, 7. To create a quantum network, quantum information is generated, processed, and stored locally in quantum nodes. These nodes are linked by quantum channels that transport quantum states from site to site with high fidelity and that distribute entanglement across the entire network. With relatively modest processing capabilities, one could even envision a 'quantum internet' that could accomplish tasks that are otherwise impossible within the realm of classical physics, including the distribution of 'quantum software' 8, 9.

Apart from any particular algorithm, there is an important advantage to be gained from quantum as opposed to classical connectivity between nodes [10. A network of quantum nodes linked by classical channels comprised of $k$ nodes each with $n$ qubits has a state space of dimension $k 2^{n}$, whereas a fully quantum network linked by quantum channels has an exponentially larger dimension $2^{k n}$. Quantum connectivity also provides a potentially powerful means to overcome size-scaling and error-correlation problems that would otherwise limit the size of machines for quantum processing [11. At any stage in the development of quantum technologies, there will be a largest size attainable for individual quantum processing units, which can be superseded by linking such units together into a fully quantum network.

A quite different perspective of a quantum network is to view the nodes as components of a physical system that interact by way of the quantum channels. In this case, the underlying physical processes used for quantum network protocols are adapted to simulate the evolution of quantum many-body systems [12. For example, atoms localized at separate nodes can have effective "spin - spin" interactions catalyzed by single-photon pulses that travel along the channels between the nodes [13. The "quantum wiring" of the network allows a wide range for the effective Hamiltonian and topology of the resulting "lattice". Moreover, the extension of entanglement across quantum networks can be related to the classical problem of percolation [14].

These exciting opportunities motivate an examination of research related to the actual physical processes for translating the abstract illustration in Fig. 1(a) into reality. From diverse activities worldwide, I will narrow the focus to provide an overview of current efforts and prospects for harnessing optical processes at the level of single photons and atoms for the reliable transport of quantum states across complex quantum networks. In this context, we require that quantum states of a material system be mapped to and from propagating optical fields as illustrated in Fig. 1 (b), thereby achieving quantum connectivity for the network, including entanglement to enable quantum teleportation between nodes.

Such considerations are timely since scientific capabilities are now passing the threshold from a learning phase with individual systems over the past fifteen years and are advancing into a domain of rudimentary functionality for 


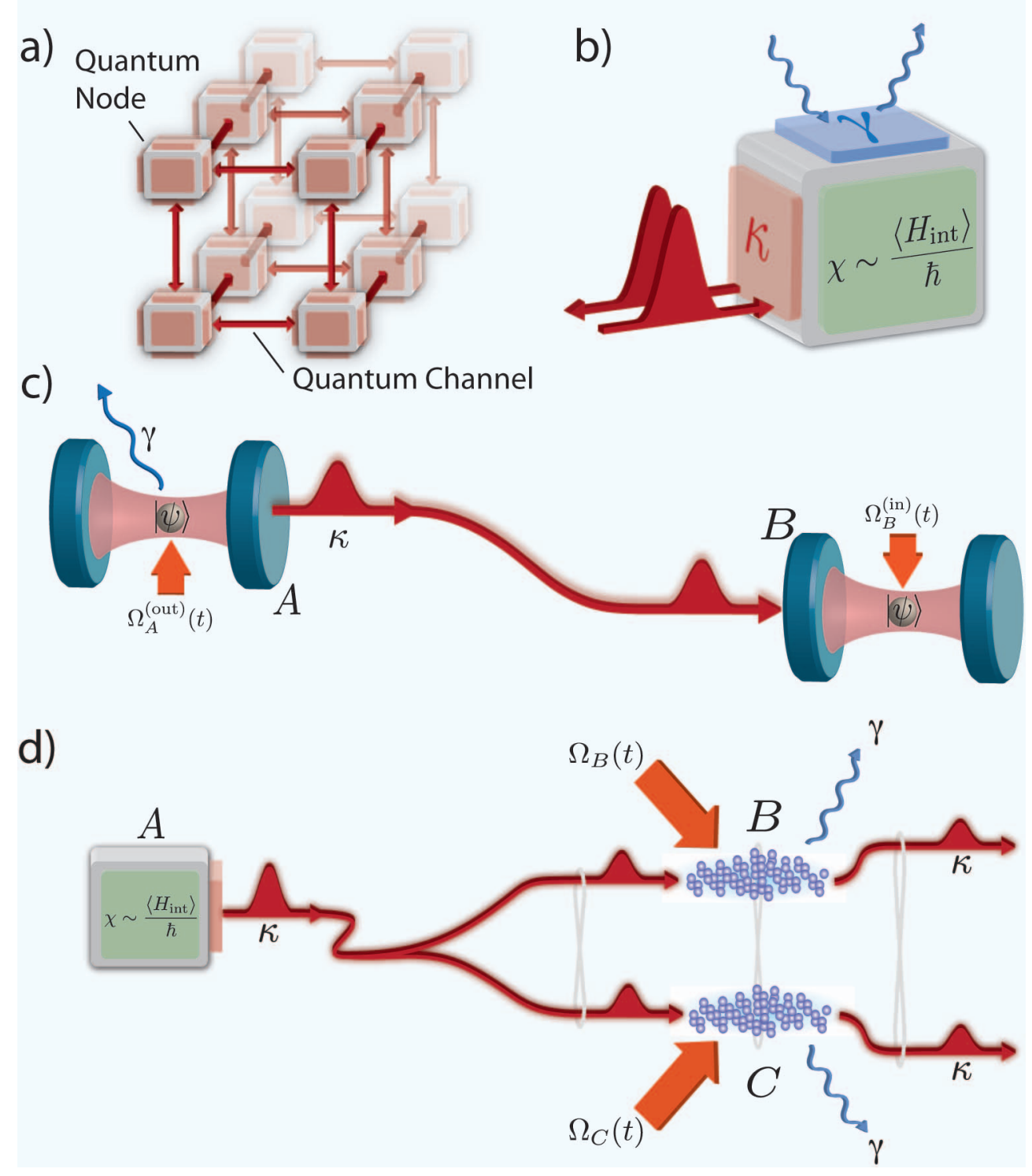

FIG. 1: Quantum networks from abstract to physical. (a) A quantum network composed of quantum nodes for processing and storing quantum states and quantum channels for the distribution of quantum information. Such a network can alternatively be viewed as a strongly correlated many-particle system. (b) Quantum interface between matter and light. Coherent interactions within the node are characterized by the rate $\chi$, while $\kappa$ specifies the rate for coupling between the node and photons in the external channel. Parasitic losses occur at rate $\gamma$. (c) Quantum state transfer and entanglement distribution from node $A$ to $B$ within the setting of cavity QED [7. At node $A$ the control pulse $\Omega_{A}^{\text {out }}(t)$ affects the transformation of atomic state $|\psi\rangle$ to the state of a propagating optical field (i.e., a 'flying photon'). At node $B$ the pulse $\Omega_{B}^{i n}(t)$ is applied to map the state of the flying photon into an atom within the cavity, thereby realizing the transfer of the state $|\psi\rangle$ from $A$ to $B$ [22]. (d) Distribution of entanglement using ensembles of a large number of atoms [16. A single-photon pulse at node $A$ is coherently split into two entangled components that propagate to nodes $B, C$ and are there coherently mapped by control fields $\Omega_{B, C}^{(i n)}(t)$ into an entangled state between ensembles at $B, C$. At later times, components of the entangled state can be retrieved from the quantum memories by separate control fields $\Omega_{B, C}^{(o u t)}(t)[23$. 
quantum nodes connected by quantum channels. From a broad spectrum of remarkable achievements, two important areas on which I will focus are strong coupling of single photons and atoms within the setting of cavity quantum electrodynamics (QED) [15] and quantum information processing with atomic ensembles [16. Critical to both these examples are long-lived quantum memories provided by the atomic system and efficient light-matter interfaces.

I apologize at the outset for my sins of omission related to alternative approaches. In these early days, it is impossible to foresee which of many exciting possibilities might lead to rudimentary networks with nontrivial scientific capabilities much less to functional systems of technological significance. Many physical systems are being investigated as surveyed in Ref. [2] and described at the following websites: http://qist.lanl.gov/qcomp_map.shtml, http://www.scala-ip.org/public/, and http://www.qubitapplications.com/. However, my purpose is not to present an exhaustive set of detailed descriptions, but rather to convey basic principles for physical implementations of quantum networks by way of a few examples in Quantum Optics. I strive thereby to stimulate the involvement of a larger community in this endeavor, including for systems-level studies.

\section{A QUANTUM INTERFACE BETWEEN LIGHT AND MATTER}

The principal scientific challenge in the quest to distribute quantum states across a quantum network is the attainment of coherent control over the interactions of light and matter at the single-photon level. Whereas atoms and electrons have relatively large long-range interactions for their spin and charge degrees of freedom, individual photons have interaction cross-sections that are typically orders of magnitude too small for nontrivial dynamics when coupled to single degrees of freedom for a material system.

The endeavor to address this issue began in the optical physics community in the 1990s with the development of theoretical protocols for the coherent transfer of quantum states between atoms and photons within the setting of cavity quantum electrodynamics (QED) [7, 17, 18. Other important advances have been made in the past decade, including with atomic ensembles [16, 19, 20]. The reversible mapping of quantum states between light and matter provides the basis for quantum-optical interconnects and is a fundamental primitive for building quantum networks. Although the original schemes for such interconnects are fragile to experimental imperfections, a complete set of theoretical protocols have subsequently been developed for the robust distribution of quantum information over quantum networks, including, quite significantly, the invention of the quantum repeater [4, 21] and scalable quantum networks with atomic ensembles [16.

Fig. 1(b) illustrates a generic quantum interface between light and matter described by the interaction Hamiltonian $H_{\text {int }}(t)$, where for typical states $\left\langle H_{\text {int }}(t)\right\rangle \sim \hbar \chi(t)$, with $\chi(t)$ the time-dependent coupling strength between the internal material system and the electromagnetic field. Desiderata for a quantum interface include that $\chi(t)$ should be "user-controlled" for clocking states to and from the quantum memory (e.g., by way of an auxiliary laser), that the physical processes employed should be robust in the face of imperfections (e.g., by adiabatic transfer), and that mistakes should be efficiently detected and fixed (e.g., with quantum error correction). In qualitative terms, the rate $\kappa$ which characterizes the bandwidth of the input-output channel should be large compared to the rate $\gamma$ for any parasitic losses, and both these rates should be small compared to the rate of coherent coupling, $\chi \gg \kappa \gg \gamma$.

Two specific examples of physical systems to realize a quantum interface and distribute coherence and entanglement between nodes are shown in Fig. 1(c), (d). In the case of Fig. 1(c), single atoms are trapped within optical cavities at nodes $A, B$ which are linked by an optical fiber. External fields control the transfer of the quantum state $|\psi\rangle$ stored in the atom at node $A$ to the atom at node $B$ by way of photons that propagate from $A$ to $B$ [7, 22. In Fig. 1(d), a single photon pulse generated at node $A$ is coherently split into two components and propagates to nodes $B, C$, where the entangled photon state is coherently mapped into an entangled state between collective excitations at the two nodes [16, 19, 20, 23. Subsequent readout of entanglement from either or both of the memories at $B, C$ to photon pulses is implemented at the push of a button.

In the sections that follow, I will elaborate in somewhat more detail the underlying physical processes for the implementation of quantum-optical interconnects between matter and light. Particular attention will be given to the two examples in Fig. 11(c), (d).

\section{CAVITY QUANTUM ELECTRODYNAMICS}

At the forefront of the endeavor to achieve strong, coherent interactions between light and matter has been the area of cavity quantum electrodynamics (QED) [24. In both the optical [15, 25] and microwave [26, 27, 28, 29, 30. domains, strong coupling of single atoms and photons has been achieved by utilizing electromagnetic resonators of 
small mode volume $V_{m}$ with quality factors $Q \sim 10^{7}-10^{11}$. Extensions of cavity QED to other systems 31 include quantum dots coupled to micropillars and photonic bandgap cavities [32, 33, 34, 35] and Cooper-pairs interacting with superconducting resonators (i.e., 'circuit' QED reviewed in Ref. [36]).

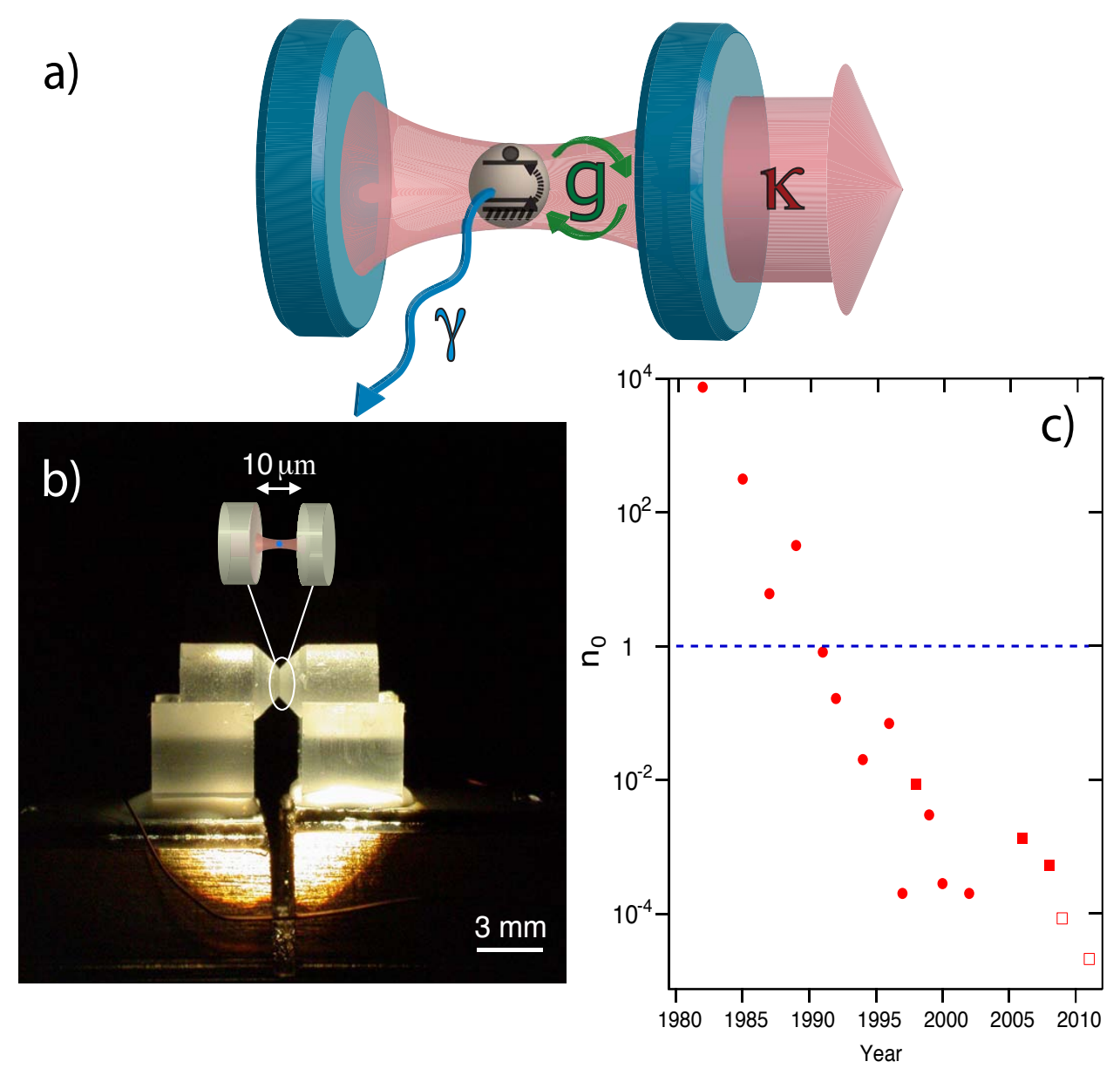

FIG. 2: Elements of cavity QED. (a) A simple schematic of an atom-cavity system depicting the three governing rates $(g, \kappa, \gamma)$ in cavity QED, where $g \sim \chi$ from Fig. 1. (b) Photograph of two mirror substrates that form a Fabry-Perot cavity shown schematically above. The cavity has length $l=10 \mu \mathrm{m}$, waist $w_{0}=12 \mu \mathrm{m}$ transverse to the cavity axis, and finesse $F \simeq 5 \times 10^{5}$. The supporting structure serves for active servo control of the cavity length to $\delta l \simeq 10^{-14} \mathrm{~m}$ [15]. (c) Critical photon number $n_{0}$ versus time for a series of experiments in cavity QED, first at the Unversity of Texas at Austin and then in the Caltech Quantum Optics Group. These experiments involve both spherical mirror Fabry-Perot cavities (circles) and the whispering gallery modes of monolithic $\mathrm{SiO}_{2}$ resonators (squares). The points in 2006,2008 are from Refs. [43, 44] demonstrated for a $\mathrm{SiO}_{2}$ microtoroidal resonator, while those in 2009, 2011 are projections for a microtoroid [45].

Figure 2(a, b) depicts a single atom located inside an optical resonator, for which strong coupling of atom and photon requires that a single intracavity photon creates a "large" electric field. Stated more quantitatively, if the coupling frequency of one atom to a single mode of an optical resonator is $g$ (i.e., $2 g$ is the one-photon Rabi frequency), then

$$
g=\sqrt{\frac{\left|\vec{\varepsilon} \cdot \vec{\mu}_{0}\right|^{2} \omega_{C}}{2 \hbar \epsilon_{0} V_{m}}} .
$$

Here, $\vec{\mu}_{0}$ is the transition-dipole moment between the relevant atomic states with transition frequency $\omega_{A}$, and $\omega_{C} \simeq \omega_{A}$ is the resonant frequency of the cavity field with polarization vector $\vec{\varepsilon}$ and mode volume $V_{m}$. Experiments 
in cavity QED explore strong coupling with $g \gg(\gamma, \kappa)$, where $\gamma$ is the atomic decay rate to modes other than the cavity mode and $\kappa$ is the decay rate of the cavity mode itself. Expressed in the language of traditional optical physics, the number of photons required to saturate the intracavity atom is $n_{0} \sim \gamma^{2} / g^{2}$, and the number of atoms required to have an appreciable effect on the intracavity field is $N_{0} \sim \kappa \gamma / g^{2}$. Strong coupling in cavity QED moves beyond traditional optical physics for which $\left(n_{0}, N_{0}\right) \gg 1$ to explore a qualitatively new regime with $\left(n_{0}, N_{0}\right) \ll 1[15$.

A variety of approaches have been employed to achieve strong coupling in cavity QED over the past three decades, with reviews of much of the early work available in Ref. 24]. Of particular note in the development of this field were the pioneering experiments of $\mathrm{H}$. Walther [26], who combined Rydberg atoms with superconducting cavities $\left(Q \sim 10^{10}\right)$ to advance cavity QED into a regime of strong coupling in the microwave domain [28, 29]. A recent spectacular achievement is the observation of the step-by-step collapse of the quantum state of an intracavity microwave field [30].

In the optical domain, a route to strong coupling is the use of high-finesse optical resonators $\left(F \sim 10^{5}-10^{6}\right)$ and atomic transitions with large $\vec{\mu}_{0}$ (i.e., oscillator strengths near unity). Figure 2 (c) provides an illustration of progress along this avenue, with research now well into the domain $\left(n_{0}, N_{0}\right) \ll 1$.

Of course as the cavity volume $V_{m}$ is reduced to increase $g$ as in Eq. 1. the requirement for atomic localization necessarily becomes more stringent. Not surprisingly, central to activities in cavity QED over the past decade have been efforts to trap and localize atoms within high-finesse optical cavities in a regime of strong coupling, with the initial demonstration in 1999 [37. Subsequent advances are reviewed in Ref. 38, including trap lifetimes $\gtrsim 10 \mathrm{~s}$ [39, 40. Quantum control has now been achieved over both internal (i.e., atomic dipole and cavity field) and external (i.e., atomic motion) degrees of freedom for a strongly coupled atom-cavity system 41. An exciting prospect is cavity QED with single trapped ions, where the boundary for strong coupling has been reached [42].

\section{Coherence and entanglement in cavity QED}

An application of these capabilities to quantum networks is the generation of single photons "on demand" (see Box 1 ). By way of strong coupling of the cavity field to an atomic transition, an external control field $\Omega(t)$ transfers one photon into the cavity mode and thence to free-space by way of the cavity output mirror, leading to a single-photon pulse $\left|\phi_{1}(t)\right\rangle$ as a collimated beam. The temporal structure (amplitude and phase) of the resulting "flying photon" $\left|\phi_{1}(t)\right\rangle$ can be tailored by way of the control field $\Omega(t)$ [7, 46, 47], with the spatial structure of the wavepacket set by the cavity mode $\psi(\vec{r})$.

Several experiments have confirmed the essential aspects of this process for the deterministic generation of single photons [39, 42, 48. Significantly, in the ideal, adiabatic limit, the atomic excited state $|e\rangle$ is not populated [49]. By deterministically generating a bit stream of single-photon pulses from single, trapped atoms, these experiments provide a first step in the evolution of quantum networks based upon flying photons.

Relative to single-photon generation by diverse other systems [50], a distinguishing aspect of the dark-state protocol discussed in Box 1 is that it should be reversible, so that a photon emitted from one system $A$ can be efficiently transferred to another system $B$ by simply applying the time-reversed (and suitably delayed) field $\Omega(t)$ at $B$, as illustrated in Figure 1(c).

Such an advance was reported in Ref. 22] by implementing the reversible mapping of a coherent optical field to and from internal states of a single, trapped Cesium atom. As described by Eq. 2 in Box 1, the incident field $\left|\phi_{\text {field }}(t)\right\rangle \sim c_{0}|0\rangle_{\text {field }}+c_{1}|1\rangle_{\text {field }}$ was approximated by a coherent state $\lambda(t)$ with $\bar{n}=1.1$ photons and was transferred into a coherent superposition of atomic states. The stored atomic state was then coherently mapped back into a propagating field $\beta(t)$. Although there were imperfections in this experiment [22, it provides the initial verification of the fundamental primitive upon which the protocol in Ref. 7] is based.

The discussion in Box 1 as well as related possibilities [13, 47] rely upon strong coupling between an atom and a single polarization of the intracavity field. However, by extending these ideas to the two polarization eigenmodes of the cavity for a given $\mathrm{TEM}_{00}$ longitudinal mode, it is possible to generate entanglement between internal atomic states and the polarization state of a coherently generated photon [51, 52, 53]. An initial control field $\Omega_{1}\left(t_{1}\right)$ results in an entangled state between internal states of the atom $\left|b_{ \pm}\right\rangle$and the polarization state of a flying photon $\left|\phi_{\text {field }}^{ \pm}\left(t_{1}\right)\right\rangle$ coherently generated by the coupled atom-cavity system. Application of a second control field $\Omega_{2}\left(t_{2}\right)$ returns the atom to its initial (unentangled) state while generating a second flying photon $\left|\xi_{\text {field }}^{ \pm}\left(t_{2}\right)\right\rangle$, thereby leading to entanglement between the polarizations $\sigma_{ \pm}$of the two fields $\phi_{\text {field }}^{ \pm}, \xi_{\text {field }}^{ \pm}$emitted at times $t_{1}, t_{2}$.

Precisely such a sequence of operations $\Omega_{1,2}\left(t_{1,2}\right)$ has been applied to single Rubidium atoms falling through a highfinesse optical cavity [25]. In an experimental tour de force, these authors were able to create entangled photons with time separation $\tau=t_{2}-t_{1}$ limited by the atomic transit time. Although the atoms arrived randomly, the protocol 
itself is intrinsically deterministic and represents a significant advance toward the generation and distribution of entangled states for quantum networking. With trapped atoms, it will be possible to generate entangled states at user selected times $\left(t_{1}, t_{2}\right)$ at the "push of button." Moreover, the scheme is inherently reversible, so that the entangled state between atom and field can be used to distribute entanglement to a second atom-cavity system in a network.

In a broader context, important advances have been made in the generation and transfer of quantum states with other physical systems. For example, single photons generated by a quantum dot coupled to a photonic-bandgap cavity have been transmitted to a second, 'target' cavity via an on-chip waveguide [54. Ref. [36] reviews the spectacular progress made in wiring quantum circuits by way of superconducting microwave cavities and coherent transmission along a quantum bus.

This discussion provides a preview of coming experimental advances towards the realization of quantum networks based upon interactions in cavity QED. Diverse theoretical protocols have been developed but have awaited the maturation of experimental capabilities, which we are now witnessing. Included are the sequential generation of entangled multiqubit states [55, the teleportation of atomic states from one node to another [18, photonic quantum computation by photon-photon interactions at the nodes [47, and reversible mapping of quantum states of atomic motion to and from light [56. Certainly, new technical capabilities beyond conventional Fabry-Perot cavities will be required to enable such scientific investigations, with Box 2 discussing some candidate systems.

\section{QUANTUM NETWORKS WITH ATOMIC ENSEMBLES}

An area of considerable activity in the quest to distribute coherence and entanglement across quantum networks has been the interaction of light with atomic ensembles comprised of a large collection of identical atoms. For the regime of continuous variables, entanglement has been achieved between two atomic ensembles each consisting of $\sim 10^{12}$ atoms [67, and quantum teleportation of light to matter demonstrated for the mapping of coherent optical states to the collective spin states of an atomic memory [68. More general activities in this area are covered in Ref. 69.

Here, I will focus instead on the regime of discrete variables with photons and atomic excitations taken one by one. Research in this area is based upon the remarkable theoretical protocol in Ref. [16] (hereafter referred to as 'DLCZ'), which presented a realistic scheme for entanglement distribution by way of a quantum-repeater architecture [4, 21.

Fundamental to the DLCZ protocol is the generation and retrieval of single 'spin' excitations within an ensemble of a large number of atoms (see Box 3) [70. In concert with photoelectric detection, a first laser pulse creates a single excitation $\left|1_{a}\right\rangle$ stored collectively within the atomic ensemble. At a later time, a second pulse deterministically converts excitation stored within the atomic memory in the state $\left|1_{a}\right\rangle$ into a propagating field, denoted as field 2.

The basic processes illustrated in Box 3 can be extended to create an entangled pair of ensembles as in Fig. 3(a) [16]. A pair of ensembles $L, R$ is illuminated by separate write pulses, leading to the overall state $\left|\phi_{L, R}\right\rangle=\left|\phi_{a, 1}^{L}\right\rangle \otimes\left|\phi_{a, 1}^{R}\right\rangle$, where $\left|\phi_{a, 1}^{L}\right\rangle,\left|\phi_{a, 1}^{R}\right\rangle$ are entangled states between atomic excitation and photon number for field 1 for the $L, R$ ensembles (see Eq. 1 in Box 3). The scattered fields $1_{L}, 1_{R}$ from the two ensembles are combined on a $50-50$ beamsplitter, with outputs directed to two photodetectors $D_{1}, D_{2}$. In the ideal case and to lowest order in $p$, a photoelectric detection event at either detector projects the ensembles into the entangled state

$$
\left|\Psi_{L, R}\right\rangle=\frac{1}{\sqrt{2}}\left[\left|0_{a}\right\rangle_{L}\left|1_{a}\right\rangle_{R} \pm e^{i \eta_{1}}\left|1_{a}\right\rangle_{L}\left|0_{a}\right\rangle_{R}\right]
$$

with the sign + or - set by whether $D_{1}$ or $D_{2}$ records the event. The phase $\eta_{1}$ is determined by the difference of phase shifts along the two channels, $\eta_{1}=\beta_{L}-\beta_{R}$ [71, which must be stable.

The state $\left|\Psi_{L, R}\right\rangle$ is generated in a probabilistic but heralded fashion from quantum interference in the measurement process [72, 73, 74]. That is, detection of a photon from one or the other atomic ensemble in an indistinguishable fashion results in an entangled state with one collective spin excitation shared coherently between the ensembles. Necessarily, because $p \ll 1$, any given trial with the write pulses is unlikely to produce a detection event at $D_{1}, D_{2}$, with such failed trials requiring the system to be reinitialized. However, a photoelectric detection event at $D_{1}, D_{2}$ unambiguously heralds the creation of the entangled state $\left|\Psi_{L, R}\right\rangle$. Limited by the coherence time between the levels $\left\{|g\rangle_{i},|s\rangle_{i}\right\}$ [75], this entangled state is stored in the quantum memory provided by the $L, R$ ensembles and available 'on demand' for subsequent tasks, such as entanglement connection [16, 76].

Although the preceding discussion is for an ideal case and neglects higher-order terms, the DLCZ protocol is designed to be resilient to important sources of imperfections, including losses in propagation and detection, as well as detector dark counts. Indeed, the scheme functions with "built-in entanglement purification" [16] and enables entanglement to be extended beyond the separation of two ensembles in an efficient and scalable fashion. Theoretical extensions 

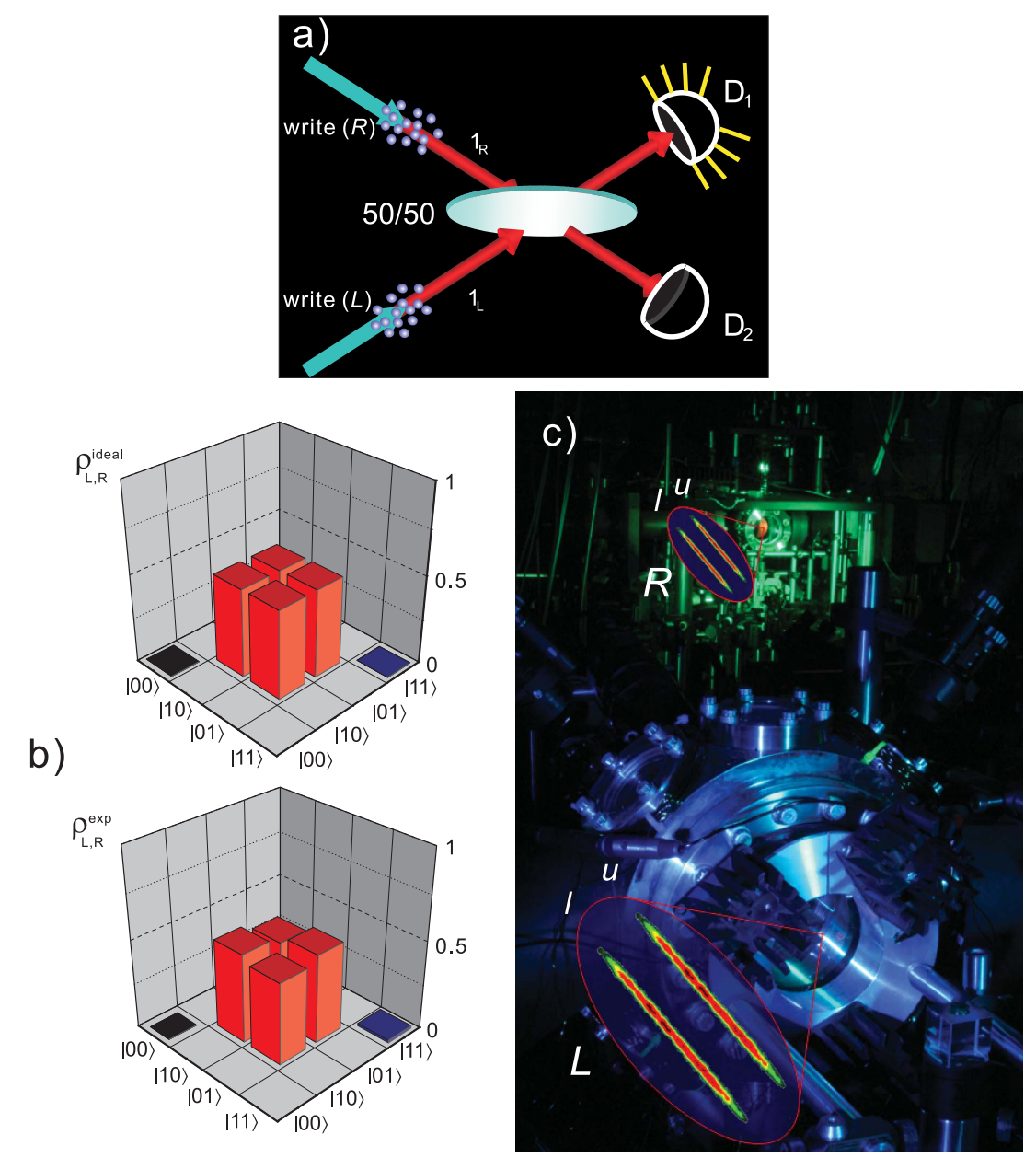

FIG. 3: Fundamentals of the DLCZ protocol [16]. a) Measurement-induced entanglement between two atomic ensembles $L, R$ [16. 71. Synchronized laser pulses incident on the ensembles (write beams $L, R$ ) generate small amplitudes for spontaneous Raman scattering [70, denoted as $1_{L}, 1_{R}$. These fields interfere at a 50\%-50\% beam splitter, with outputs directed to singlephoton detectors $D_{1}, D_{2}$. A measurement event at either detector projects the $L, R$ ensembles into the entangled state $\left|\Psi_{L, R}\right\rangle$ with one quantum of excitation shared remotely between the ensembles. Entanglement is stored in the quantum memory provided by the ensembles and can be subsequently converted to propagating light pulses by a set of read laser pulses (Box 3). b) Experimentally determined elements of the density matrix $\rho_{L, R}^{\exp }$ for entanglement between two atomic ensembles 75 , corresponding to concurrence $C=0.9 \pm 0.3$, where $C=0$ for an unentangled state. For comparison the density matrix $\rho_{L, R}^{t h}$ for the ideal state $\left|\Psi_{L, R}\right\rangle$ is shown with concurrence $C=1$. c) Photograph of the laboratory setup for entanglement of two pairs of atomic ensembles for the realization of functional quantum nodes $L, R$ separated by $3 \mathrm{~m}$ 93. The four elongated ovals each show a cylinder of $10^{5}$ Cesium atoms which forms an atomic ensemble at each site. Entangled states $\left|\Psi_{L, R}^{u}\right\rangle,\left|\Psi_{L, R}^{l}\right\rangle$ between the upper $u$ and lower $l$ pairs at the $L, R$ nodes are generated and stored in an asynchronous fashion for each pair $u, l$ as in a). Atomic excitations for the pairs $L_{u}, L_{l}$ and $R_{u}, R_{l}$ are subsequently converted to flying photons at each node with polarization encoding that leads to violation of a Bell inequality [93]. The entire experiment functions under the quantum control of single detection events.

[77, 78, 79] of the DLCZ protocol have examined related network architectures for optimizing scalability in view of actual laboratory capabilities, to which I next turn.

\section{Coherence and entanglement with atomic ensembles}

The publication of the DLCZ protocol in 2001 led to the rapid development of a worldwide community with by now significant achievements in the creation and distribution of entanglement. The initial, enabling steps in the implementation of the protocol of DLCZ were the observations of quantum correlations both for single photon pairs 
[81, 82 and for large photon number $\left(10^{3}-10^{4}\right)$ 83. generated in the collective emission from atomic ensembles. Single photons were generated by the efficient mapping of stored, collective atomic excitation to propagating wavepackets for field 2, as in Box 3 [84, 85, 87, 88, 89. Conditional readout efficiencies of $74 \%$ in free space [86, and 84\% 90] in a cavity were realized for the state transfer from a single, collective "spin" excitation stored in the atomic ensemble to a single photon for field 2 .

With these capabilities for coherent control of collective atomic emission, heralded entanglement between distant ensembles was achieved in 2005 [71], with the entangled state stored in quantum memories (i.e., the two ensembles) located in distinct apparatuses separated by $3 \mathrm{~m}$. After a programmable delay, entanglement was confirmed by mapping the state of the atoms to optical fields and by measuring mutual coherence and photon statistics for these fields. Although the degree of entanglement between the ensembles was small in the initial experiment [71, more recent work [75] has led to the inference $C=0.9 \pm 0.3$ for the concurrence $C$ [91] of entanglement stored between $L, R$ ensembles, with the associated density matrix $\rho_{L, R}$ shown in Fig. 3(b).

The DLCZ protocol is based upon a quantum repeater architecture involving independent operations on parallel chains of quantum systems [16, with scalability relying critically upon conditional control of quantum states stored in remote quantum memories 92. The experiment shown in Fig. 3.(c) 93] took an important step towards this goal by achieving the minimal functionality required for scalable quantum networks. Heralded entanglement was created asynchronously between a qubit located at node $L$ and one at node $R$, where each qubit was encoded by a pair of upper $u$ and lower $l$ atomic ensembles. The entangled states at the $(L, R)$ nodes were stored in the quantum memory provided by the ensembles, and then efficiently mapped to propagating light fields resulting in an effective polarization entangled state, as verified by the measured violation of a Bell inequality for these light fields. This setup was also used for the initial investigation of entanglement swapping [76] to demonstrate coherence between ensembles that had no direct interaction.

Beyond DLCZ's proposal for measurement-induced entanglement, it is also possible to achieve deterministic mapping of quantum states of light to and from atomic ensembles by way of electromagnetically induced transparency (EIT) [19, 20, 80. The pioneering work in Refs. 94, 95] demonstrated the storage and retrieval of classical pulses to and from an ensemble. Refs. [96, 97] extended this work into the quantum regime of single photons. Entanglement between two ensembles coupled to a cavity mode was achieved in Ref. 98, by adiabatic transfer of excitation, thereby providing a means for on-demand entanglement. To assist the distribution of entanglement over quantum networks, Ref. 23] realized the reversible mapping of photonic entanglement into and out of pairs of quantum memories by an EIT process illustrated in Fig. 1(d).

Contemporary with these experiments which achieved heralded [16, 71, 75, 93, and deterministic [23, 98, entanglement have been a variety of experiments based upon entanglement as a post diction [99], for which a physical state is not available for utilization in a scalable network but which are nonetheless significant. An important advance in this regard is the work in Ref. [100], which employed a pair of ensembles for entanglement generation to achieve $a$ posteriori teleportation of light to an atomic memory.

There has also been considerable effort devoted to the detailed characterization of decoherence for stored atomic excitation and entanglement [75, 93, 100]. Decoherence of entanglement between distinct atomic ensembles was observed in the decay of the violation of a Bell inequality [93] and of the fidelity for teleportation [100. By way of measurements of concurrence $C(t)$, Ref. [75] presented quantitative characterizations for the relationship of the global evolution of the entangled state to the temporal dynamics of various local correlations.

\section{EXTENDING ENTANGLEMENT FOR QUANTUM NETWORKS}

The entangled states created so far both in cavity QED and by way of the DLCZ protocol are between pairs of systems, so-called bipartite entanglement, for which definitive procedures exist for operational verification. Certainly the creation of more general classes of entangled states shared among $N>2$ systems would be of great interest. In this regard, an important area of ongoing research is the development of theoretical protocols amenable to laboratory implementation for entanglement verification of quantum states distributed over the nodes of a quantum network. However, as we progress toward more complex quantum networks, the issue of entanglement verification becomes increasingly problematic. Theoretical tools and experimental capabilities do not at present exist for characterizing the general states of quantum networks.

Perhaps surprisingly, a nontrivial task will be simply to answer the question "Does it work?" That is, as even moderately complex quantum networks are realized in the laboratory, it will become ever more difficult to assess quantitatively the characteristics of the network, including such basic questions as "Does entanglement extend across the whole network?" On the one hand, we might follow a strategy motivated by the underlying physical processes 
upon which the network is based and attempt to determine, for example, the density matrix $\rho(t)$ for the network. This course of action surely fails because of the exponential growth in $\rho(t)$ with the size of the network.

We might instead follow a strategy based upon more functional issues of algorithmic capability. That is, we could attempt to implement a quantum algorithm for computation or communication to test whether the purported quantum network actually accomplishes anything beyond the capabilities of any classical counterpart. This course is problematic since the advantage of a quantum network may only be realized above some threshold in the size of the network. Furthermore, from an experimental perspective, this strategy does not offer much in the way of diagnostics for 'fixing' the network when it fails.

A less obvious approach might be to adopt more seriously the perspective of a quantum network as a quantum many body system and to search for more 'physical' characteristics of the network (e.g., the scaling behavior of pair correlation functions and multipartite entanglement). Indeed, an active area of research is the nature of entanglement for systems that undergo quantum phase transitions, including the pioneering advance for 1-D spin chains in Ref. [101.

\section{CONCLUSION}

Although important progress has been made, there remain many scientific challenges that require new theoretical insights and experimental capabilities in the quest to realize functional quantum networks. Certainly, the current state of the art is primitive relative to that required for the robust and scalable implementation of sophisticated network protocols, whether over short or long distances. The realization of quantum memories, local quantum processing, quantum repeaters, and error-corrected teleportation are very ambitious goals. There is nevertheless considerable activity worldwide directed towards these important goals.

While my discussion has considered separately cavity QED-based networks and those implemented via the DLCZ protocol, clearly quantum networks will evolve as heterogeneous entities. For example, the same protocol that creates the entangled state $\left|\Psi_{L, R}\right\rangle$ between two ensembles in Eq. 2 can be employed to create the entangled state

$$
\left|\Pi_{A, E}\right\rangle \sim\left[\left|1_{A}\right\rangle\left|0_{E}\right\rangle \pm\left|0_{A}\right\rangle\left|1_{E}\right\rangle\right],
$$

with one excitation shared jointly by an atom in a cavity $\left|1_{A}\right\rangle$ and an atomic ensemble $\left|1_{E}\right\rangle$. A critical task is the development of unambiguous procedures for entanglement verification, which is a nontrivial undertaking that has not always been carried out correctly [99].

I have used quantum networks as unifying theme but stress that the research described has much broader import, including for advancing our understanding of quantum dynamical systems and, for the particular cases considered, creating new physics from controlled nonlinear interactions of single photons and atoms. Altogether, these are exciting times in Quantum Information Science as we pass from a regime of individual 'Lego blocks' (e.g., a single atom-cavity system) into the realm of complex quantum systems assembled from many such units from the ground up. One important area of this scientific enterprise is the realization of quantum networks. I have every confidence that extending entanglement across quantum networks will create wonderful scientific opportunities for the exploration of physical systems that have not heretofore existed in the natural world. 
[1] Nielsen, M. A. \& Chuang, I. L. Quantum Computation and Quantum Information. (Cambridge University Press, 2000).

[2] For a recent review of progress, see Zoller, P. et al. Quantum information processing and communication, Strategic report on current status, visions and goals for research in Europe. Eur. Phys. J. D 36, 203-228 (2005).

[3] Bennett, C. H., Brassard, G. \& Ekert, A. K. Quantum cryptography. Scientific American 267(4), 50-57 (1992).

[4] Briegel, H. -J., van Enk, S. J., Cirac, J. I. \& Zoller, P. in The Physics of Quantum Information (eds. Bouwmeester, D., Ekert, A. \& Zeilinger, A.) (Springer, Berlin, 2000).

[5] Spiller, T. P. et al. Quantum computation by communication New J. Phys. 8, (27 February 2006).

[6] Giovannetti, V., Lloyd, S. \& Maccone, L. Quantum-enhanced measurements: Beating the standard quantum limit. Science 306, 1330-1336 (2004).

[7] Cirac, J. I., Zoller, P., Kimble, H. J. \& Mabuchi, H. Quantum state transfer and entanglement distribution among distant nodes in a quantum network. Phys. Rev. Lett. 78, 3221-3224 (1997).

[8] Preskill, J. P. Plug-in Quantum Software. Nature 402, 357-358 (1999).

[9] Gottesman, D. \& Chuang, I. Demonstrating the viability of universal quantum computation using teleportation and single-qubit operations. Nature 402, 390-393 (1999).

[10] Cirac, J. I., Ekert, A. K., Huelga, S. F. \& Macchiavello, C. Distributed quantum computation over noisy channels Phys. Rev. A 59, 4249-4254 (1999).

[11] Copsey, D. et al. Toward a scalable, silicon-based quantum computing architecture. IEEE J. Selected Topics in Quantum Electronics 9, 1552-1569 (2003).

[12] Illuminati, D. Quantum optics: Light does matter. Nature Phys. 2, 803-804 (2006) and references therein.

[13] Duan, L. -M., Wang, B. \& Kimble, H. J. Robust quantum gates on neutral atoms with cavity-assisted photon scattering. Phys. Rev. A 72, 032333 (2005).

[14] Acín, A., Cirac, J. I. \& Lewenstein, M., Entanglement percolation in quantum networks. Nature Physics 3, 256-259 (2007).

[15] Miller, R., Northup, T. E., Birnbaum, K. M., Boca, A., Boozer, A. D. \& Kimble, H. J. Trapped atoms in cavity QED: coupling quantized light and matter. J. Phys. B: At. Mol. Opt. Phys. 38, S551-S565 (2005).

[16] Duan, L. -M., Lukin, M. D., Cirac, J. I. \& Zoller, P. Long-distance quantum communication with atomic ensembles and linear optics. Nature 414, 413-418 (2001).

[17] Parkins, A. S., Marte, P., Zoller, P. \& Kimble, H. J. Synthesis of arbitrary quantum states via adiabatic transfer of Zeeman coherence. Phys. Rev. Lett. 71, 3095-3098 (1993).

[18] van Enk, S. J., Cirac, J. I. \& Zoller, P. Photonic channels for quantum communication. Science 279, 205-208 (1998).

[19] Lukin, M. D. Colloquium: Trapping and manipulating photon states in atomic ensembles. Rev. Mod. Phys. 75, 457-472 (2003).

[20] Fleischhauer, M., Imamoglu, A. \& Marangos, J. P. Electromagnetically induced transparency: Optics in coherent media. Rev. Mod. Phys. 77, 633-673 (2005).

[21] Briegel, H. -J., Dür, W., Cirac, J. I. \& Zoller, P. Quantum Repeaters: The role of imperfect local operations in quantum communication. Phys. Rev. Lett. 81, 5932-5935 (1998).

[22] Boozer, A. D., Boca, A., Miller, R., Northup, T. E. \& Kimble, H. J. Reversible state transfer between light and a single trapped atom. Phys. Rev. Lett. 98, 193601 (2007).

[23] Choi, K. S., Deng, H., Laurat, J. \& Kimble, H. J. Mapping photonic entanglement into and out of a quantum memory. Nature 452, 67-71 (2008).

[24] Berman, P. (ed.) Cavity Quantum Electrodynamics, (Academic Press, San Diego, 1994).

[25] Wilk, T., Webster, S. C., Kuhn, A. \& Rempe, G. Single-atom single-photon quantum interface. Science 317, 488-490 (2007).

[26] Meschede, D., Walther, H. \& Mueller, G. One-atom maser. Phys. Rev. Lett. 54, 551-554 (1985).

[27] Meystre, P. Cavity Quantum Optics and the Quantum Measurement Process. in Progress in Optics, Vol. XXX (ed. E. Wolf) (Elsevier Science Publishers B.V., Amsterdam, 1992).

[28] Walther, H. Fortschr. Phys. Quantum optics of single atoms. 52, 1154-1164 (2004).

[29] Raimond, J. M. et al. Probing a quantum field in a photon box. J. Phys. B: At. Mol. Opt. Phys. 38, S535-S550 (2005).

[30] Guerlin, C. et al. Progressive field-state collapse and quantum non-demolition photon counting. Nature 448, 889-893 (2007).

[31] For a review, see Vahala, K. J. Optical microcavities. Nature 424, 839-846 (2004).

[32] For a review, see Khitrova, G., Gibbs, H. M., Kira, M., Koch, S. W. \& Scherer, A. Vacuum Rabi splitting in semiconductors. Nature Physics 2, 81-90 (2006).

[33] Srinivasan, K. \& Painter, O. Linear and nonlinear optical spectroscopy of a strongly coupled microdisk-quantum dot system. Nature 450, 862-865 (2007).

[34] For a review, see A. J. Shields, Nature Photonics 1, 215 (2007).

[35] Englund, D. et al. Controlling cavity reflectivity with a single quantum dot. Nature 450, 857-861 (2007).

[36] Schoelkopf, R. J. \& Girvin, S. M. Wiring up quantum systems. Nature 451, 664-669 (2008).

[37] Ye, J., Vernooy, D. W. \& Kimble, H. J. Trapping of single atoms in cavity QED. Phys. Rev. Lett. 83, $4987-4990$ (1999).

[38] For a review of optical traps in cavity QED, see Ye, J., Kimble, H. J. \& Katori, H. Quantum state engineering and precision metrology using state-insensitive light traps. Science (in press, 2008). 
[39] Hijlkema, M. et al. A single-photon server with just one atom. Nature Physics 3, 253-255 (2007).

[40] Fortier, K. M., Kim, S. Y., Gibbons, M. J. Ahmadi, P. \& Chapman, M. S. Deterministic loading of individual atoms to a high-finesse optical cavity. Phys. Rev. Lett. 98, 233601 (2007).

[41] Boozer, A. D., Boca, A., Miller, R., Northup, T. E. \& Kimble, H. J. Cooling to the ground state of axial motion for one atom strongly coupled to an optical cavity. Phys. Rev. Lett. 97, 083602 (2006).

[42] Keller, M., Lange, B., Hayasaka, K., Lange, W. \& Walther, H. Continuous generation of single photons with controlled waveform in an ion-trap cavity system. Nature 431, 1075-1078 (2004).

[43] Aoki, T., Dayan, B., Wilcut, E., Bowen, W. P., Parkins, A. S., Kimble, H. J., Kippenberg, T. J. \& Vahala, K. J. Observation of strong coupling between one atom and a monolithic microresonator. Nature 443, 671-674 (2006).

[44] Dayan, B., Parkins, A. S., Aoki, T., Ostby, E. P., Vahala, K. J. \& Kimble, H. J. A photon turnstile dynamically regulated by one atom. Science 319, 1062-1065 (2008).

[45] Spillane, S. M., Kippenberg, T. J., Vahala, K. J., Goh, K. W., Wilcut, E. \& Kimble, H. J. Ultrahigh-Q toroidal microresonators for cavity quantum electrodynamics. Phys. Rev. A 71, 013817 (2005).

[46] Kuhn, A., Hennrich, M., Bondo, T. \& Rempe, G. Controlled Generation of Single Photons from a Strongly Coupled Atom-Cavity System. Appl. Phys. B 69, 373-377 (1999).

[47] Duan, L. -M. \& Kimble, H. J. Scalable photonic quantum computation through cavity-assisted interactions. Phys. Rev. Lett. 92, 127902 (2004).

[48] McKeever, J., Boca, A., Boozer, A. D., Miller, R., Buck, J. R., Kuzmich, A. \& Kimble, H. J. Deterministic generation of single photons from one atom trapped in a cavity. Science 303, 1992-1994 (2004).

[49] Bergmann, K., Theuer, H. \& Shore, B. W.Coherent population transfer among quantum states of atoms and molecules, Rev. Mod. Phys. 70, 1003-1025 (1998).

[50] Lounis, B. \& Orrit, M. Single-photon sources. Rep. Prog. Phys. 68, 1129-1179 (2005).

[51] Lange, W. \& Kimble, H. J. Dynamic generation of maximally entangled photon multiplets by adiabatic passage. Phys. Rev. A 61, 063817 (2000).

[52] Duan, L. -M. \& Kimble, H. J. Efficient engineering of multiatom entanglement through single-photon detections. Phys. Rev. Lett. 90, 253601 (2003).

[53] Sun, B., Chapman, M. S. \& You, L. Atom-photon entanglement generation and distribution. Phys. Rev. A 69, 042316 (2004).

[54] Englund, D., Faraon, A., Zhang, B., Yamamoto, Y. \& Vučković, J. Generation and transfer of single photons on a photonic crystal chip. Opt. Express 15, 5550-5558 (2007).

[55] Schön, C., Solano, E., Verstraete, F., Cirac, J. I. \& Wolf, M. M. Sequential generation of entangled multiqubit states. Phys. Rev. Lett. 95, 110503 (2005).

[56] Parkins, A. S. \& Kimble, H. J. Quantum state transfer between motion and light. Journal Opt. B: Quantum Semiclass. Opt. 1, 496-504 (1999).

[57] Hood, C. J., Ye, J. \& Kimble, H. J. Characterization of high-finesse mirrors: Loss, phase shifts, and mode structure in an optical cavity. Phys. Rev. A 64, 033804 (2001).

[58] Vernooy, D. W., Furusawa, A., Georgiades, N. Ph., Ilchenko, V. S. \& Kimble, H. J. Cavity QED with high-Q whispering gallery modes. Phys. Rev. A 57, R2293-R2296 (1998).

[59] Trupke, M. et al. Atom detection and photon production in a scalable, open, optical microcavity. Phys. Rev. Lett. 99, 063601 (2007).

[60] Treutlein, P. et al. Quantum information processing in optical lattices and magnetic microtraps. Fortschritte der Physik - Progress of Physics 54, 702-718 (2006).

[61] Park, Y. -S., Cook, A. K. \& Wang, H. Cavity QED with diamond nanocrystals and silica microspheres. Nano Letters 6, 2075-2079 (2006).

[62] Xu, X. et al. Coherent Optical Spectroscopy of a Strongly Driven Quantum Dot. Science 317, 929-932 (2007).

[63] Armani, D. K., Kippenberg, T. J., Spillane, S. M. \& Vahala, K. J. Ultra-high-Q toroid microcavity on a chip. Nature 421, 925-928 (2003).

[64] Spillane, S. M., Kippenberg, T. J., Painter, O. J. \& Vahala, K. J. Ideality in a fiber-taper-coupled microresonator system for application to cavity quantum electrodynamics. Phys. Rev. Lett. 91, 043902 (2003).

[65] Braginsky, V. B., Gorodetsky, M. L. \& Ilchenko, V. S. Quality-factor and nonlinear properties of optical whispering-gallery modes. Phys. Lett. A 137, 393-397 (1989).

[66] Vernooy, D. W., Ilchenko, V. S., Mabuchi, H., Streed, E. W. \& Kimble, H. J. High-Q measurements of fused-silica microspheres in the near infrared. Opt. Lett. 23, 247-249 (1998).

[67] Julsgaard, B., Kozhekin, A. \& Polzik, E. S. Experimental long-lived entanglement of two macroscopic objects. Nature 413, 400-403 (2001).

[68] Sherson, J. F. et al. Quantum teleportation between light and matter. Nature 443, 557-560 (2006).

[69] For a review, see Cerf, N. J., Leuchs, G. \& Polzik, E. S. (eds.) Quantum Information with Continuous Variables (World Scientific Publishing, New Jersey, 2007).

[70] Raymer, M. G., Walmsley, I. A., Mostowski, J. \& Sobolewska, B. Quantum theory of spatial and temporal coherence properties of stimulated Raman scattering. Phys. Rev. A 32, 332-344 (1985).

[71] Chou, C. -W., de Riedmatten, H., Felinto, D., Polyakov, S. V., van Enk, S. J. \& Kimble, H. J. Measurement-induced entanglement for excitation stored in remote atomic ensembles. Nature 438, 828-832 (2005).

[72] Dicke, R. H. Interaction-free quantum measurements: A paradox? Am. J. Phys. 49, 925-930 (1981).

[73] Cabrillo, C., Cirac, J. I., García-Fernández, P. \& Zoller, P. et al. Creation of entangled states of distant atoms by 
interference. Phys. Rev. A 59, 1025-1033 (1999).

[74] Bose, S. et al. Proposal for Teleportation of an Atomic State via Cavity Decay Phys. Rev. Lett. 83, 5158 - 5161 (1999).

[75] Laurat, J., Choi, K. S., Deng, H., Chou, C.-W. \& Kimble, H. J. Heralded entanglement between atomic ensembles: Preparation, decoherence, and scaling. Phys. Rev. Lett. 99, 180504 (2007).

[76] Laurat, J. et al. Towards experimental entanglement connection with atomic ensembles in the single excitation regime. New J. Phys. 9, 207-220 (2007).

[77] Jiang, L., Taylor, J. M. \& Lukin, M. D. Fast and robust approach to long-distance quantum communication with atomic ensembles. Phys. Rev. A 76, 012301 (2007).

[78] Zhao, B., Chen, Z. -B., Chen, Y. -A., Schmiedmayer, J \& Pan, J. -W. Robust creation of entanglement between remote memory qubits. Phys. Rev. Lett. 98, 240502 (2007).

[79] Sangouard, N. et al., Robust and efficient quantum repeaters with atomic ensembles and linear optics, available at http://arxiv.org/abs/0802.1475.

[80] Harris, S. E. Electromagnetically induced transparency. Phys. Today 50, 36-40 (1997).

[81] Kuzmich, A., Bowen, W. P., Boozer, A. D., Boca, A., Chou, C. W., Duan, L. -M. \& Kimble, H. J. Generation of nonclassical photon pairs for scalable quantum communication with atomic ensembles. Nature 423, $731-734$ (2003).

[82] Balić, V., Braje, D. A., Kolchin, P., Yin, G. Y. \& Harris, S. E. Generation of paired photons with controllable waveforms. Phys. Rev. Lett. 94, 183601 (2005).

[83] van der Wal, C. H., Eisaman, M. D., André, A., Walsworth, R. L., Phillips, D. F., Zibrov, A. S. \& Lukin, M. D. Atomic memory for correlated photon states. Science 301, 196 (2003).

[84] Chou, C. W., Polyakov, S. V., Kuzmich, A. \& Kimble, H. J. Single-photon generation from stored excitation in an atomic ensemble. Phys. Rev. Lett. 92, 213601 (2004).

[85] Laurat, J. et al. Efficient retrieval of a single excitation stored in an atomic ensemble. Opt. Express 14, 6912-6918 (2006).

[86] Du, S. et al. Subnatural linewidth biphotons with controllable temporal length. Phys. Rev. Lett. 100, 18603 (2006).

[87] Thompson, J. K., Simon, J., Loh, H. \& Vuletić, V. A high-brightness source of narrowband, identical-photon pairs. Science 313, 74-77 (2006).

[88] Matsukevich, D. N. et al. Deterministic single photons via conditional quantum evolution. Phys. Rev. Lett. 97, 013601 (2006).

[89] Chen, S. et al. Deterministic and storable single-photon source based on a quantum memory. Phys. Rev. Lett. 97, 173004 (2006).

[90] Simon, J., Tanji, H., Thompson, J. K. \& Vuletić, V. Interfacing collective atomic excitations and single photons. Phys.Rev. Lett. 98, 183601 (2007).

[91] Wootters, W. K. Entanglement of formation of an arbitrary state of two qubits. Phys. Rev. Lett. 80, 2245-2248 (1998).

[92] Felinto, D., Chou, C. W., Laurat, J., Schomburg, E. W., de Riedmatten, H. \& Kimble, H. J., Conditional control of the quantum states of remote atomic memories for quantum networking, Nature Physics 2, 844-848 (2006) (advanced online publication 29 October, 2006).

[93] Chou, C. -W., Laurat, J., Deng, H., Choi, K. S., de Riedmatten, H., Felinto, D. \& Kimble, H. J. Functional quantum nodes for entanglement distribution over scalable quantum networks. Science 316, 1316-1320 (2007); published online 5 April 2007 (DOI 10.1126/science.1140300).

[94] Liu, C., Dutton, Z., Behroozi, C. H. \& Hau, L. V. Observation of coherent optical information storage in an atomic medium using halted light pulses. Nature 409, 490-493 (2001).

[95] Phillips, D. F., Fleischhauer, A., Mair, A., Walsworth, R. L. \& Lukin, M. D. Storage of light in atomic vapor. Phys. Rev. Lett. 86, 783-786 (2001).

[96] Chanelière, T. et al. Storage and retrieval of single photons transmitted between remote quantum memories. Nature 438, 833-836 (2005).

[97] Eisaman, M. D. et al. Electromagnetically induced transparency with tunable single-photon pulses. Nature 438, 837-841 (2005).

[98] Simon, J., Tanji, H., Ghosh, S. \& Vuletić, V. Single-photon bus connecting spin-wave quantum memories. Nature Physics 3, 765-769 (2007); advance online publication, 23 September 2007 (DOI 10.1038/nphys726).

[99] van Enk, S. J., Lütkenhaus, N. \& Kimble, H. J. Experimental procedures for entanglement verification. Phys. Rev. A 75, 052318 (2007).

[100] Chen, Y. -A. et al. Memory-built-in quantum teleportation with photonic and atomic qubits. Nature Physics 4, 103-107 (2008).

[101] Vidal, G., Latorre, J. I., Rico, E., Kitaev, A., Entanglement in quantum critical phenomena. Phys. Rev. Lett. 90, 227902 (2003).

Acknowledgement - I gratefully acknowledge the contributions made by members of the Caltech Quantum Optics Group, and especially K. S. Choi, B. Dayan, and R. Miller for their efforts on this manuscript. I am indebted to J. P. Preskill and S. J. van Enk for their insights. This research is supported by the National Science Foundation, by IARPA, and by Northrop Grumman Space Technology.

Correspondence - Correspondence and requests for materials should be addressed to HJK. (email: hjkimble@caltech.edu). 


\section{BOX 1 - MAPPING QUANTUM STATES BETWEEN ATOMS AND PHOTONS}

Reversible state transfer between light and a single trapped atom can be achieved by way of the mappings $|b\rangle|1\rangle \rightarrow$ $|a\rangle|0\rangle$ and $|a\rangle|0\rangle \rightarrow|b\rangle|1\rangle$ for the coherent absorption and emission of single photons as illustrated in parts (i) and (ii) of the figure [22. Here $|a\rangle,|b\rangle$ represent internal states of the atom with long-lived coherence (e.g., atomic hyperfine states in the $6 S_{1 / 2}, F=3$ and $F=4$ manifolds of atomic Cesium) and $|0\rangle,|1\rangle$ are Fock states of the intracavity field with $n=0,1$ excitations. The transition $|b\rangle \leftrightarrow|e\rangle$ is strongly coupled to a mode of an optical cavity with interaction energy $\hbar g$. In this simple setting, the interaction Hamiltonian for atom and cavity field has a "dark state" (i.e., no excited state component $|e\rangle)$ [4] given by [17]

$$
|D\rangle=\cos \theta|a\rangle|0\rangle+\sin \theta|b\rangle|1\rangle
$$

where $\cos \theta=\left[1+\frac{\Omega^{2}(t)}{g^{2}}\right]^{-1 / 2}$ with $\Omega(t)$ as a classical control field. For $\Omega(t=0)=0$, we have $|D\rangle=|a\rangle|0\rangle$, while for $\Omega(t \rightarrow \infty) \gg g,|D\rangle \rightarrow|b\rangle|1\rangle$.

In (i), by adiabatically ramping a control field $\Omega_{1}(t) \gg g$ from on to off over a time scale $\Delta t$ slow compared to $1 / g$, the atomic state is mapped from $|b\rangle$ to $|a\rangle$ with the accompanying coherent absorption of 1 intracavity photon. Conversely, in (ii) by turning a control field $\Omega_{2}(t)$ from off to on, the atomic state is mapped from $|a\rangle$ to $|b\rangle$ with the transfer of 1 photon into the cavity mode.

As shown in (a)-(c) in the figure, these two processes can be combined to achieve the coherent transfer of the state of a propagating optical field $\lambda(t)=\left|\phi_{\text {field }}(t)\right\rangle$ into and out of a quantum memory formed by the atomic states $|a\rangle,|b\rangle$ [22]. In the ideal case, the mapping is specified by

$$
\left|\phi_{\text {field }}(t)\right\rangle|b\rangle \rightarrow^{(a)}|0\rangle\left(c_{1}|a\rangle+c_{0}|b\rangle\right) \ldots{ }^{(b)} \cdots \rightarrow^{(c)}\left|\phi_{\text {field }}(t+\tau)\right\rangle|b\rangle .
$$

where the field state is taken to be a coherent superposition of zero and one photon, $\left|\phi_{\text {field }}(t)\right\rangle=\mathcal{E}(t)\left[c_{0}|0\rangle_{\text {field }}+\right.$ $\left.c_{1}|1\rangle_{\text {field }}\right]$. $\mathcal{E}(t)$ describes the envelope of the field external to the cavity, with $\int|\mathcal{E}(t)|^{2} d t=1$. Given timing information for incoming field $\left|\phi_{\text {field }}(t)\right\rangle$, step (a) in this process is accomplished by adiabatically ramping the control field $\Omega_{1}(t)$ from on to off. In (b), the internal states of the atom provide a long-lived quantum memory. At a user-selected later time $t+\tau$, step (c) is initiated by turning $\Omega_{2}(t+\tau)$ from off to on, thereby coherently mapping the atomic state $c_{1}|a\rangle+c_{0}|b\rangle$ back to the "flying" field state $\beta(t)=\left|\phi_{\text {field }}(t+\tau)\right\rangle$.

\section{a) Light-to-Atom}

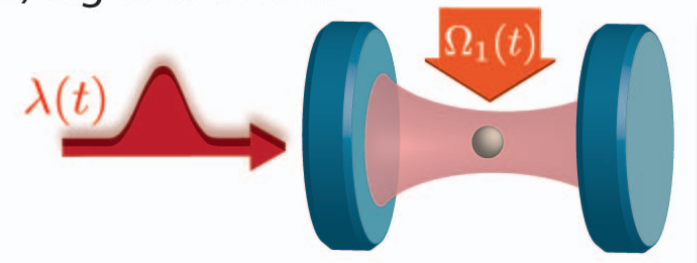

b) Quantum Memory

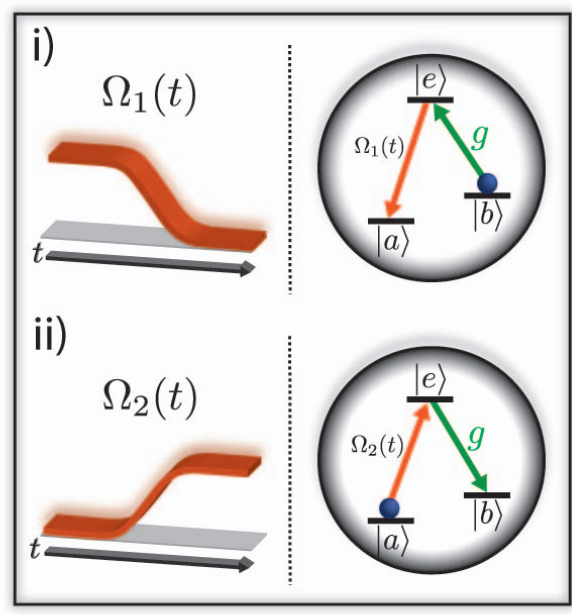

c) Atom-to-Light
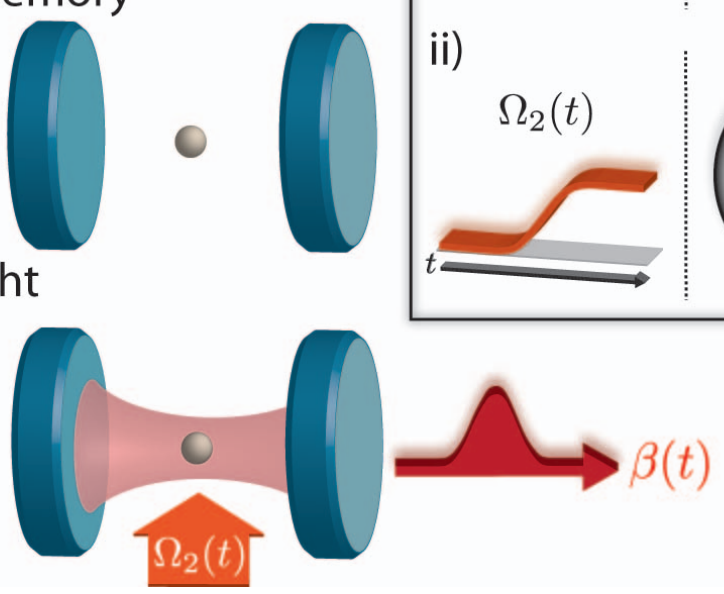


\section{BOX 2 - A NEW PARADIGM FOR CAVITY QED}

The realization of large-scale quantum networks [4, 7] requires the capability to interconnect many quantum nodes over quantum channels, for which conventional Fabry-Perot configurations are ill suited [57. There have correspondingly been efforts to develop of alternative microcavity systems [31, 58, both for single atoms [43, 44, 59, 60] and atom-like systems [61, 62, such as nitrogen vacancy centers in diamond 61. A quantitative comparison of many candidate systems is provided by Table I in Ref. [45.

A remarkable resonator for this purpose is the microtoroidal cavity formed from fused silica $\left(\mathrm{SiO}_{2}\right)$ shown in the figure 63, 64. Such a resonator supports a whispering gallery mode (WGM) 65 circulating around the outer circumference of the toroid with an evanescent field external to the resonator, as shown in part (c) of the figure. Because of the small mode volume $V_{m}$ and large quality factor $Q$ [65, 66], an atom interacting with the evanescent field of a WGM can be well into the regime of strong coupling with projected values for the critical photon $n_{0}$ and atom $N_{0}$ numbers $\left(n_{0}, N_{0}\right) \simeq\left(2 \times 10^{-5}, 10^{-6}\right)$ [45, respectively, significantly beyond current [15] and projected [45, 57] capabilities for cavity QED with Fabry-Perot cavities (Fig. 2(c)).

The fabrication techniques pioneered in Refs. 63, 64] lend themselves to the integration of many microtoroidal resonators to form optical networks, as illustrated in (a) and (b) of the figure. Part (a) shows a photograph of silicon chip with a linear array of microtoroidal resonators within a UHV apparatus [44. The toroids appear as small scattering centers on a silicon chip that runs vertically down the center of the picture. The black arrows point to a horizontal $\mathrm{SiO}_{2}$ fiber taper for coupling light to and from one resonator. Part (b) is a SEM micrograph of an array of microtoroidal resonators showing toroids of fused silica on silicon supports 63 .

These resonators have the capability for input-output coupling with small parasitic loss 64 for the configuration shown in (d), which is a photograph of an individual toroid and fiber coupler from (a) [44. Quality factors $Q=4 \times 10^{8}$ have been realized at $\lambda=1550 \mathrm{~nm}$ and $Q \simeq 10^{8}$ at $\lambda=850 \mathrm{~nm}$, with good prospects for improvement to $Q \sim 10^{10}$ [45]. For these parameters, the efficiency $\epsilon$ for coupling quantum fields into and out of the resonator could approach $\epsilon \sim 0.99-0.999$, while still remaining firmly in the regime of strong coupling [45. Such high efficiency is critical for the realization of complex quantum networks, including for the distribution and processing of quantum information [4, 7, 47] and for investigations of the association between quantum many-body systems and quantum networks [12, 14].

The initial step in this quest was the demonstration of strong coupling between individual atoms and the field of a microtoroidal resonator in Ref. 43. More recently, nonclassical fields have been generated from the interaction of single atoms with a microtoroidal resonator by way of a 'photon turnstile' for which a single atom dynamically regulates the transport of photons one-by-one through the microtoroidal resonator [44, as illustrated in (d). Only single photons can be transmitted in the forward direction (to the left in the figure), with excess photons $n>1$ dynamically rerouted to the backward direction.

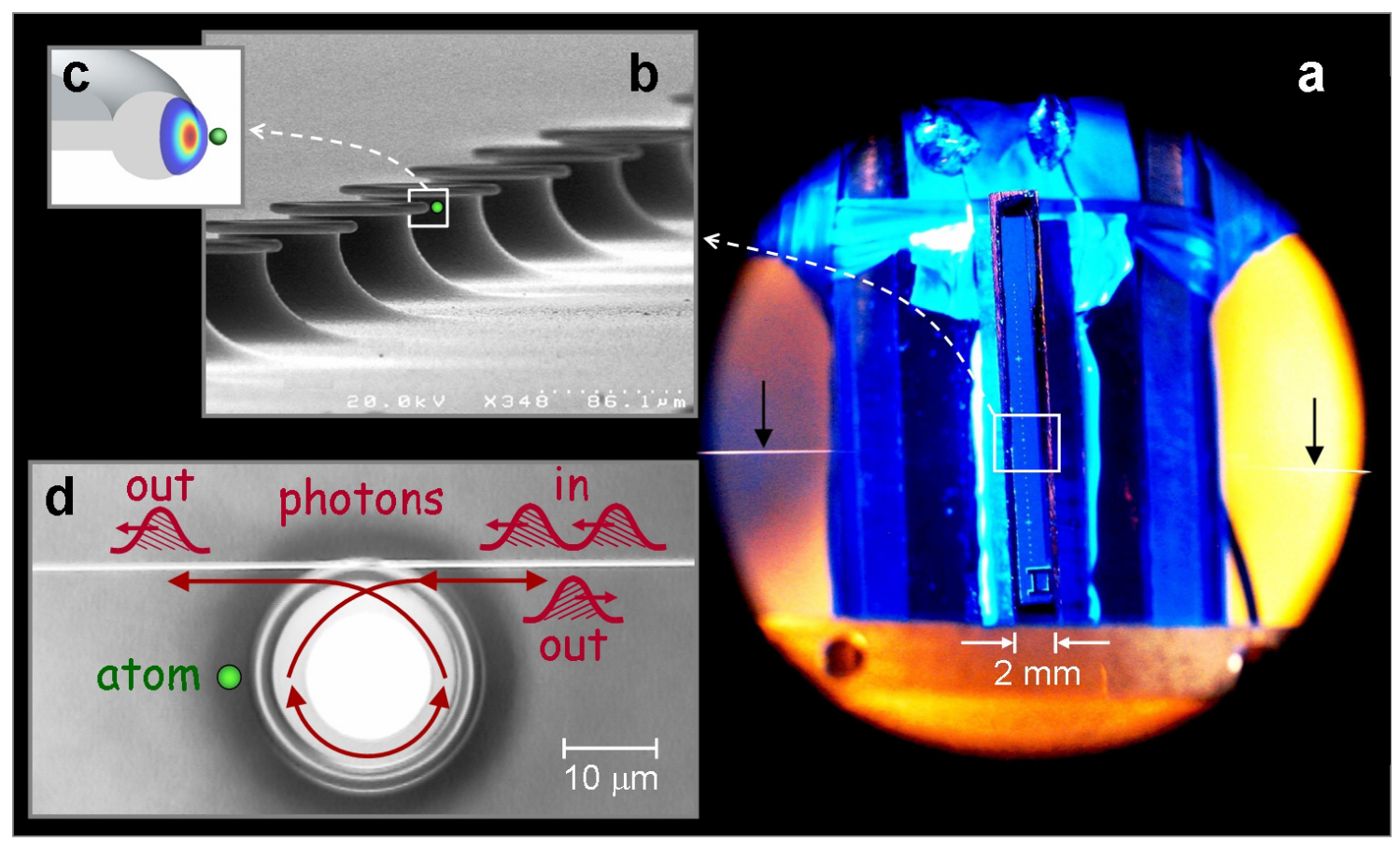




\section{BOX 3 - WRITING AND READING SINGLE ATOMIC EXCITATIONS}

The DLCZ protocol [16] is based upon ensembles of $N_{a}$ identical atoms with a $\Lambda$-level configuration as shown in the figure. The metastable lower states $|g\rangle$ and $|s\rangle$ can be, e.g., atomic hyperfine states of the electronic ground level to ensure a long lifetime for coherence. As illustrated in (a), all atoms are initially prepared in state $|g\rangle$ with no excitation, namely $\left|0_{a}\right\rangle \equiv \otimes_{i}^{N_{a}}|g\rangle_{i}$, and a weak off-resonant 'write' pulse is then sent through the ensemble. There results a small amplitude $\sqrt{p}$ for one of the $N_{a}$ atoms to be transferred from $|g\rangle$ to $|s\rangle$ and to emit a photon into the forward-scattered optical mode (designated as field 1) with frequency and/or polarization distinct from the write field.

Of course for small excitation probability $p \ll 1$, mostly nothing happens as a result of the writing pulse, so that the resulting state $\left|\phi_{a, 1}\right\rangle$ for the atomic ensemble and field 1 in the ideal case is given by

$$
\left|\phi_{a, 1}\right\rangle=\left|0_{a}\right\rangle\left|0_{1}\right\rangle+e^{i \beta} \sqrt{p}\left|1_{a}\right\rangle\left|1_{1}\right\rangle+O(p),
$$

where $\left|n_{1}\right\rangle$ is the state of the forward-propagating field 1 with $n_{1}$ photons and the phase $\beta$ is determined by the write pulse and field 1 propagation phases. The atomic state $\left|1_{a}\right\rangle$ in Eq. 1 is a collective (entangled) state with one excitation shared symmetrically among the $N_{a}$ atoms (i.e., one 'spin flip'), where in the ideal case [16]

$$
\left|1_{a}\right\rangle=\frac{1}{\sqrt{N_{a}}} \sum_{i=1}^{N_{a}}|g\rangle_{1} \cdots|s\rangle_{i} \cdots|g\rangle_{N_{a}} .
$$

Field 1 is directed to a single-photon detector, where a detection event is recorded with probability $\propto p$. Such an event for field 1 heralds that a single excitation (or spin flip $|g\rangle \rightarrow|s\rangle$ ) has been created and stored in the atomic ensemble in the state $\left|1_{a}\right\rangle$ with high probability. Higher-order processes with multiple atomic and field 1 excitations are also possible and ideally occur to lowest order with probability $p^{2}$.

After a user-defined delay (subject to the finite lifetime of the quantum memory), the collective atomic excitation $\left|1_{a}\right\rangle$ can be efficiently converted to a propagating beam (designated as field 2) by way of a strong 'read' pulse as in (b), where in the ideal case, there is a one-to-one transformation of atomic to field excitation, $\left|1_{a}\right\rangle \rightarrow\left|1_{2}\right\rangle$. For the case of resonance with the $|s\rangle \rightarrow|e\rangle$ transition, the reading process utilizes the phenomenon of electromagnetically induced transparency [19, 20, 80,
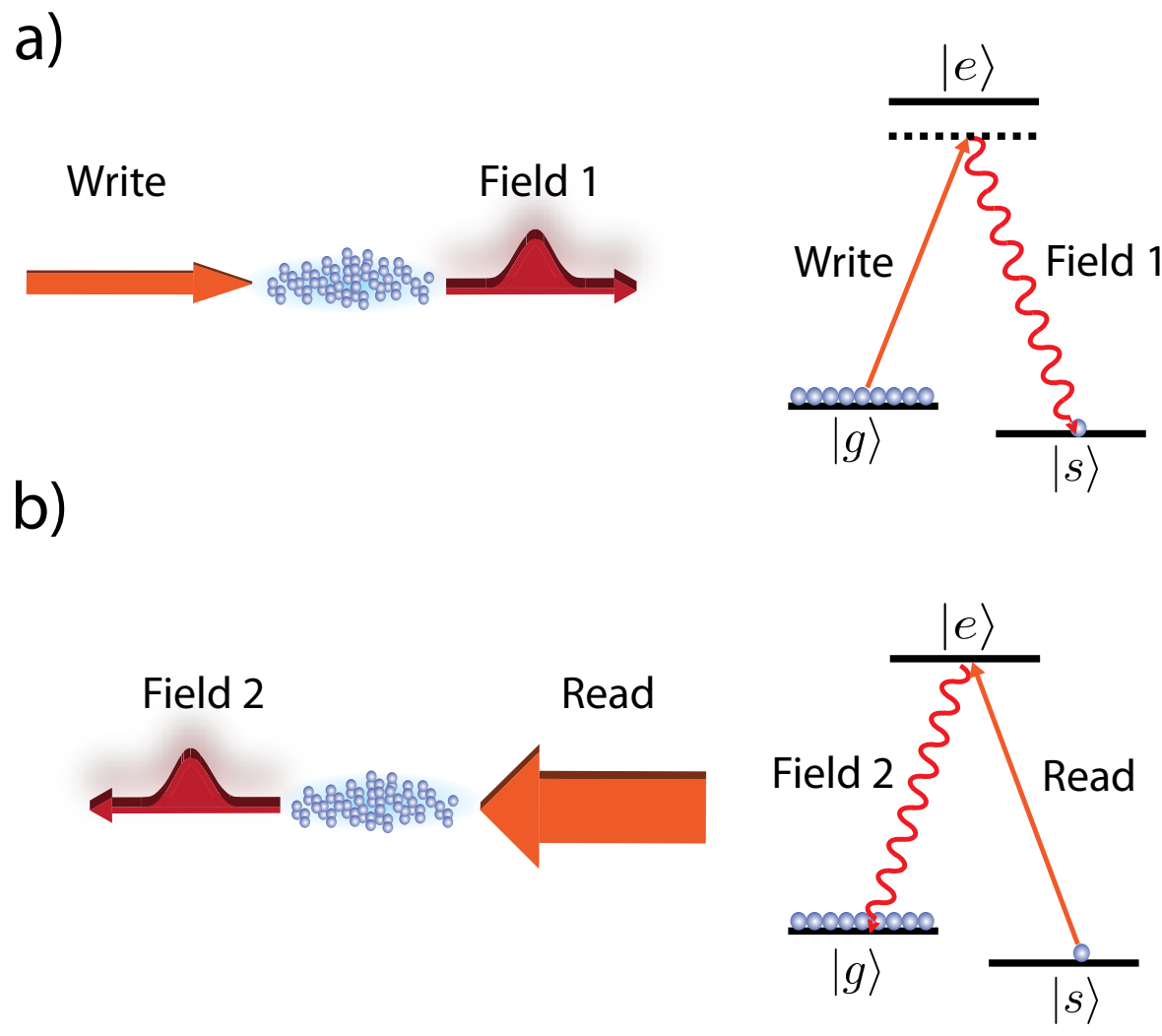